\title{
The effects of suction on forced convection boundary layer stagnation point slip flow in a Darcy porous medium towards a shrinking sheet with presence of thermal radiation: a stability analysis
}

\begin{abstract}
The stagnation-point flow in a Darcy porous medium toward a shrinking sheet with the presence of thermal radiation, suction, velocity, and thermal slips is numerically studied. The partial differentiation equations have been transformed into ordinary differentiation equations by a similarity transformation. Numerical results show the existence of dual solutions in a certain range of the governing parameters. A stability analysis to identify which solutions are stable and physically realizable by using the bvp4c solver implemented in Matlab has also been presented.
\end{abstract}

Keyword: Forced convection flow; Darcy porous medium; Shrinking sheet; Stability analysis 